\title{
Comparison of the Pain-relieving Effects of Human Milk, Sucrose, and Distilled Water during Examinations for Retinopathy of Prematurity: A Randomized Controlled Trial
}

\author{
Eun Kyung Jang ${ }^{1}$, Hyejung Lee ${ }^{2}$, Keum Sik Jo ${ }^{3}$, Sung Mi Lee ${ }^{3}$, Hyun Jin Seo ${ }^{3}$ Eun Joo Huh ${ }^{3}$ \\ ${ }^{1}$ Assistant Director, Department of Neonatology, Neonatal Intensive Care Unit, Severance Hospital, Seoul; \\ ${ }^{2}$ Associate Professor, College of Nursing · Mo-Im Kim Nursing Research Institute, Yonsei University, Seoul; \\ ${ }^{3}$ Registered Nurse, Department of Neonatology, Neonatal Intensive Care Unit, Severance Hospital, Seoul, Korea
}

Purpose: This study compared the pain-relieving effects of human milk, sucrose, and distilled water during examinations for retinopathy of prematurity. Methods: Forty-five preterm infants were randomly assigned to receive a pacifier dipped in one of three solutions: human milk $(\mathrm{n}=14), 24 \%$ sucrose $(\mathrm{n}=15)$, or distilled water $(\mathrm{n}=16), 2$ minutes before an eye examination. Their pain score, pulse rate, and oxygen saturation were measured at three time points: 5 minutes before the examination, 30 seconds after speculum introduction, and 2 minutes after the examination. Results: The infants' mean gestational age and weight at birth were $33.1 \pm 2.1$ weeks and $1,842 \pm 470 \mathrm{~g}$, respectively. There were no between-group differences in pain relief during the eye examination. The pain score significantly increased both during $(p<.001)$ and after the examinations $(p=.003)$. Oxygen saturation decreased during the examinations $(p<.001)$; however, the infants in the $24 \%$ sucrose group showed higher oxygen saturation $(p=.047)$ during the examinations than the infants in the other groups. Conclusion: Sucking on a pacifier dipped in human milk or $24 \%$ sucrose did not reduce the pain associated with eye examinations in preterm infants. Pacifiers dipped in sucrose can be used to maintain better oxygen saturation during these examinations.

Key words: Analgesia; Human milk; Pain; Retinopathy of prematurity; Sucrose

\section{Corresponding author Hyejung Lee https://orcid.org/0000-0001-9357-0640}

College of Nursing, Yonsei University, 50-1 Yonsei-ro, Sedaemun-gu, Seoul 03722, Korea

TEL +82-2-2228-3345 FAX +82-2-2227-8303

E-MAIL hlee26@yuhs.ac

Received Feb 12, 2019 Revised May 15, 2019 Accepted Jul 5, 2019 (a) This is an Open Access article distributed under the terms of the Creative Commons Attribution NonCommercial License (http://creativecommons.org/licenses/by-nc/4.0/) which permits unrestricted noncommercial use, distribution, and reproduction in any medium, provided the original work is properly cited.

\section{INTRODUCTION}

In the neonatal intensive care unit (NICU), preterm infants are subjected to various painful procedures, such as heel pricks, intravenous catheter insertion, venipuncture, and examinations for retinopathy of prematurity (ROP) [1,2]. ROP examinations are recommended for preterm infants with a birth weight between 1,500 $\mathrm{g}$ and 2,000 $\mathrm{g}$, and infants of a gestational age greater than 30 weeks who are believed to be at high risk for ROP. In the United States, ROP is the second leading cause of childhood blindness [3]; thus, ROP screening examinations are recommended. The first examination is performed at $4 \sim 6$ weeks of postnatal age or at 31 weeks of post-gestational age. However, multiple aspects of the ROP examination appear to cause discomfort and pain, including the application of dilating drops, the pressure applied to the eyeballs, and the brightness of the ophthalmoscope [4,5]. Therefore, topical anesthetics, such as proparacaine $\mathrm{HCl}(0.5 \%)$ eye drops, are commonly administered in many clinical settings to alleviate the pain induced by eye examinations [3].

In addition to pharmacological pain management, a number of non-pharmacological pain management strategies have 
been investigated to alleviate the pain associated with ROP examinations in preterm infants, including swaddling, nonnutritive sucking, and administration of sucrose or glucose solutions in varying concentrations, alone or combined $[4,6]$. Among these solutions, $0.1 \sim 2.0 \mathrm{~mL}$ of $24 \%$ sucrose has been most extensively tested in randomized controlled trials on preterm infants undergoing ROP examinations and other painful procedures, such as heel pricks, venipuncture, and intratracheal suctioning [7]. A recent study found that a minimum amount of $0.1 \mathrm{~mL}$ of $24 \%$ sucrose was effective in reducing pain in preterm and newborn infants undergoing a single heel lance $[7,8]$. In another recent study, $0.5 \mathrm{~mL}$ of $24 \%$ sucrose demonstrated better pain-relieving effects than 0.2 $\mathrm{mL}$ of the same solution in infants at a gestational age of 28 weeks and weight $>1,000 \mathrm{~g}$ undergoing venipuncture [9]. However, these studies employed painful, tissue-damaging procedures that are quite short-lasting. In contrast, ROP examinations take several minutes and involve several aspects that can cause pain that is not induced by tissue damage. Thus, the minimum amount of $24 \%$ sucrose solution required for effective pain relief in preterm infants undergoing ROP examinations remains unknown $[1,2,10]$.

The pain-relieving effects of human milk, which contains natural and potentially risk-free nutrients, have also been investigated in preterm infants in response to painful procedures [11-13]. Human milk is believed to be a relatively safe solution as opposed to sucrose, the repeated administration of which can induce hyperglycemia in younger preterm infants [14]. The pain-relieving effects of human milk have been compared with those of distilled water or sucrose in preterm infants undergoing venipuncture and ROP examinations [11,15, 16]. The composite pain scores of preterm infants who received $2 \mathrm{~mL}$ of human milk were lower than those in control group infants who received none, and this effect persisted for 5 minutes after the examination [12]. The pain responses were comparable to those in infants receiving $1 \mathrm{~mL}$ of $33 \%$ sucrose, as measured by heart rate, crying time, and salivary cortisol levels [11]. The pain-relieving effects of human milk, sucrose, and distilled water (control) during ROP examinations were also compared. No differences were found in the pain score, heart rate, or oxygen saturation between the groups $[13,16]$, although the pain scores returned to the baseline level more quickly in infants administered human milk than in those administered sucrose [16]. However, in these studies, human milk, sucrose, or distilled water was administered using an injector or a syringe without a needle, and varying $(0.1 \sim 2.0 \mathrm{~mL})$ amounts of solution were provided. Administration of a large quantity of liquid directly into the infants' mouths can potentially cause choking due to the sudden-onset crying caused by the painful procedure. Even though such instances are rare, the minimum amount of solution that is effective for pain relief should be identified. Both swaddling and the provision of a pacifier to infants are commonly practiced during ROP examinations in the NICU, and multiple non-pharmacological management strategies have been recommended in practice [17]. The purpose of this study was to compare the pain-relieving effects of human milk, $24 \%$ sucrose, and distilled water by providing a pacifier dipped in one of these solutions to preterm infants during ROP examinations.

\section{METHODS}

\section{Study Design}

A randomized controlled trial was employed to compare the pain-relieving effects of human milk, sucrose, and distilled water on preterm infants during examinations for ROP.

\section{Participants and Randomization}

This study was carried out in a 46-bed, level III NICU at an academic medical center in Seoul, Korea. The inclusion criteria were preterm infants $(<35$ weeks of gestational age at birth) whose first eye examination was scheduled. Preterm infants with a congenital anomaly and those on respiratory support or receiving analgesic drug treatment were excluded.

A total of 51 preterm infants met the eligibility criteria during the study period. Of those, 45 preterm infants were included in this study. The primary reason for non-enrollment was failure to obtain consent $(n=3)$, and the other reasons for exclusion were the absence of available human milk $(n=2)$ or not being dilated $(n=1)$ (Figure 1). The research nurse randomly allocated the 45 eligible preterm infants to one of three groups: human milk $(n=14), 24 \%$ sucrose $(n=15)$, or distilled water (control group) $(\mathrm{n}=16)$. The group assignment was carried out using a random digit table and kept in sealed envelopes when parental consent was obtained.

\section{Sample Size}

The sample size calculation was based on a previously published study of pain in infants during ROP examinations using the pain composite scale [16]. That study showed a standard deviation (SD) of 2.41 in the difference in the scores across three groups. For a given difference in the Premature Infant Pain Profile (PIPP) score of 1.02 and an SD of 2.41, a minimum total sample size of 42 preterm infants was calculated to achieve a power of $80 \%$ with a significance level of .05 , using repeated-measures analysis of variance with three betweengroup factors. 


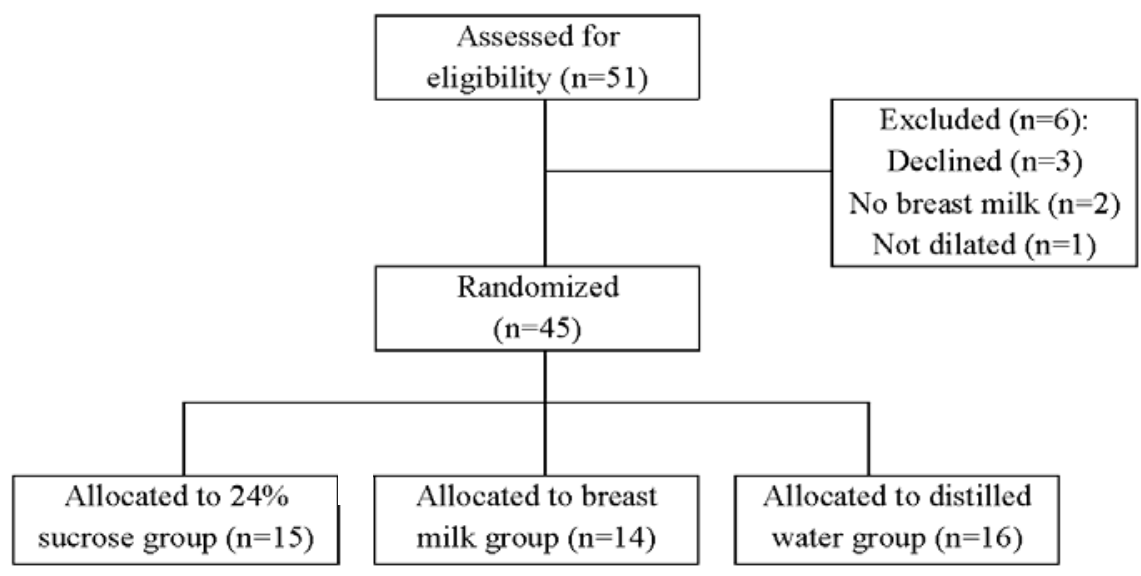

Figure 1. Flow diagram of participant recruitment.

\section{Ethical Considerations}

The study was approved by the Institutional Review Board of Severance hospital prior to data collection (Y4-2015-0442). The mothers of the eligible infants signed an informed consent form after detailed information on the research protocol was provided.

\section{Outcome Measures}

The primary outcome measure of this study was the composite pain score assessed by the PIPP [18]. The PIPP is a validated scale for the assessment of procedural pain in preterm and full-term infants based on seven indicators: gestational age, behavioral status, highest measured heart rate, lowest measured oxygen saturation, wrinkling of the forehead, squinting, and expansion of the alae nasi. The total scores range from 0 (no pain) to 21 (severe pain); higher scores indicate more pain and discomfort. A nurse educator in the NICU, who had experience in using the PIPP, trained nurse observers, who were experienced neonatal care nurses, to assess the pain of preterm infants during ROP examinations in this study. The two nurse observers independently assessed the infants' PIPP scores in real time by sitting next to the infants' beds. These nurses were blinded to the type of solution administered to each infant because the research nurse prepared the pacifier by dipping it in one of the three solutions, which were all almost colorless. For data analysis, the PIPP scores determined by the two nurse observers were averaged at each of the three time points.

The secondary outcome measures included pulse rate (PR) and oxygen saturation, which were measured by a sensor wrapped around the right foot and connected to a pulse oximetry monitor (Nellcore; Medtronic, Minneapolis, MN, USA). $\mathrm{PR}$ and oxygen saturation were continuously recorded during each examination and the research nurse recorded these measures every 15 seconds on the data monitoring form. For data analysis, PR and oxygen saturation were also averaged over 1 minute for each time point. Sociodemographic data, such as gestational age at birth, birth weight, post-conceptional age, and Apgar scores, were obtained from the infants' electronic medical records.

\section{Data Collection and Procedure}

All infants who participated in the study were administered mydriatic eye drops $(0.5 \%$ tropicamide and $2.5 \%$ phenylephrine) 1 hour prior to the examination, and topical anesthetic eye drops (proxymetacaine $0.5 \%$, Alcaine ${ }^{\circledR} 30$ seconds prior to the introduction of the eye speculum. In addition, all infants were provided swaddling and a pacifier (GumDrop Preemie pacifier; Natus, Pleasanton, CA, USA) during the examination. The ROP examinations were performed by the ophthalmologist on duty (out of two ophthalmologists assigned to the NICU), according to the protocol for ROP examinations. The ophthalmologists were blinded to the group assignment of the infants. The average duration of the examination of both eyes was 2.8 minutes; there were no differences in the examination duration among the three groups.

The infants were provided a pacifier dipped in one of the three solutions, depending on their group assignment. The research nurse, who knew the group assignment, prepared a pacifier dipped in human milk, $24 \%$ sucrose, or distilled water. A $24 \%$ sucrose solution was prepared in the pharmacy and subsequently delivered and stored in the refrigerator in the NICU, and the human milk received from each infant's mother was thawed by the research nurse just before the examination. The research nurse handed the pacifier to the primary nurse, who was assisting in the examination process by swaddling the infant and holding him or her on the bed 2 minutes 
before the examination, and infants were allowed to suck on the pacifier throughout and after the examination. Data were obtained at three time points: 5 minutes prior to the examination (baseline), 30 seconds after the speculum was introduced in the right eye (during the examination), and 2 minutes after the speculum was removed from the left eye (after the examination). Since the same type of yellowish pacifier was used for all preterm infants and all three solutions were almost colorless, the ophthalmologists, nurse observers, and parents were blind to the identity of the solution.

\section{Data Analysis}

The data analysis was performed using SPSS version 21.0 (IBM Corp., Armonk, NY, USA). Descriptive statistics and $x^{2}$ analysis were used to test the homogeneity of the three groups. Using a generalized estimating equation (GEE) analysis, the effects of solution type on PIPP scores, PR, and oxygen satu- ration were analyzed at each time point. Potential covariatesgestational age at birth, post-conceptional age, and Apgar scores-were adjusted for each outcome measure.

\section{RESULTS}

The three study groups were homogeneous in terms of sex, gestational age at birth, birth weight, weight at examination, post-conceptional age, Apgar scores at 1 and 5 minutes, and examination duration (Table 1). The mean gestational age at birth of all preterm infants was $33.1 \pm 2.1$ weeks, and the mean post-conceptional age was $35.1 \pm 1.5$ weeks. Their mean birth weight was $1,842 \pm 470 \mathrm{~g}$, and the mean Apgar scores were $4.8 \pm 1.6$ at 1 minute and $6.5 \pm 1.7$ at 5 minutes.

The mean PIPP scores, PR, and oxygen saturation at the three time points are summarized in Table 2. GEE analysis revealed no significant differences in the outcome variables

Table 1. Characteristics of the Preterm Infants in the Human Milk, Sucrose, and Distilled Water Groups

$(N=45)$

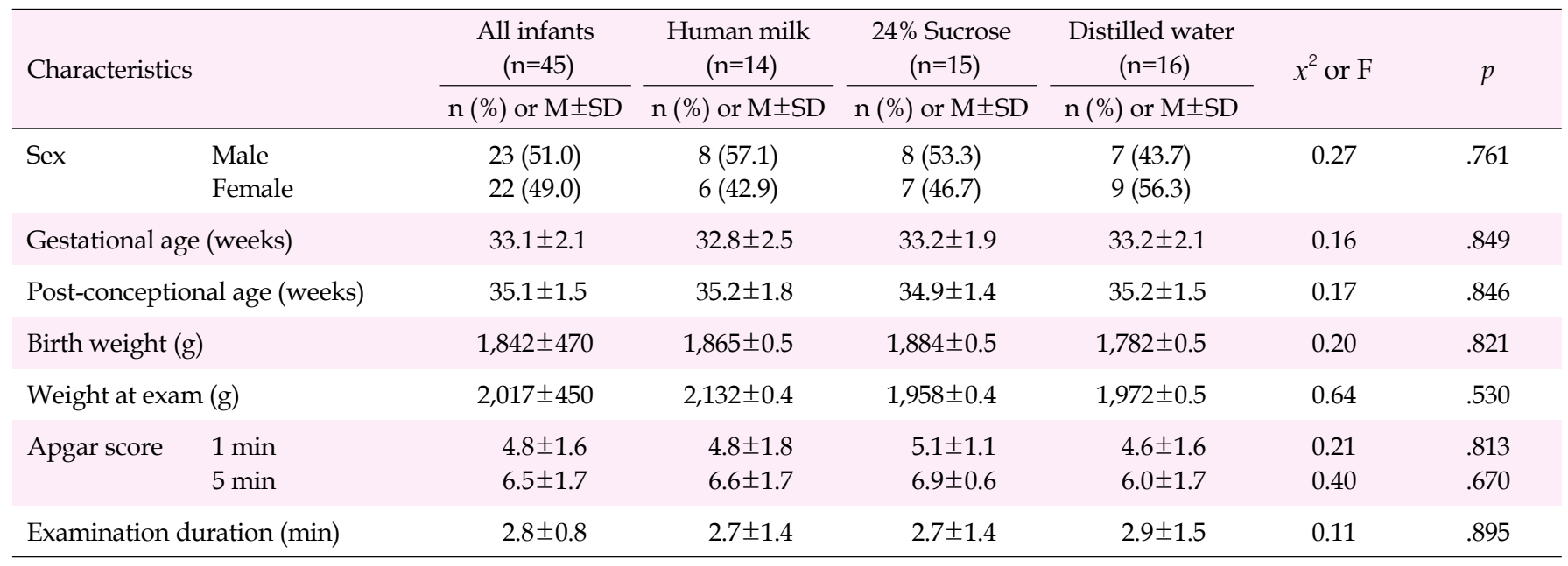

Table 2. Mean Values of Premature Infant Pain Profile Scores, Pulse Rate, and Oxygen Saturation Measured Before, During, and After Examinations for Retinopathy of Prematurity

\begin{tabular}{|c|c|c|c|c|}
\hline \multirow{2}{*}{ Group } & \multirow{2}{*}{ Time } & PIPP & PR (bpm) & Oxygen saturation (\%) \\
\hline & & $\mathrm{M} \pm \mathrm{SD}$ & $\mathrm{M} \pm \mathrm{SD}$ & $\mathrm{M} \pm \mathrm{SD}$ \\
\hline \multirow[t]{3}{*}{ Human milk (n=14) } & Before & $2.6 \pm 1.4$ & $150.4 \pm 10.1$ & $98.5 \pm 1.9$ \\
\hline & During & $14.6 \pm 3.4$ & $164.9 \pm 13.1$ & $95.7 \pm 2.8$ \\
\hline & After & $5.6 \pm 2.4$ & $164.9 \pm 19.6$ & $98.7 \pm 2.1$ \\
\hline \multirow[t]{3}{*}{$24 \%$ Sucrose $(n=15)$} & Before & $3.7 \pm 1.5$ & $152.5 \pm 13.8$ & $98.9 \pm 1.5$ \\
\hline & During & $14.7 \pm 2.7$ & $170.5 \pm 16.2$ & $97.6 \pm 2.2$ \\
\hline & After & $5.7 \pm 3.1$ & $161.1 \pm 11.8$ & $98.5 \pm 2.9$ \\
\hline \multirow[t]{3}{*}{ Distilled water $(n=16)$} & Before & $3.0 \pm 1.8$ & $151.8 \pm 11.4$ & $98.8 \pm 1.9$ \\
\hline & During & $13.8 \pm 3.7$ & $166.3 \pm 16.8$ & $95.1 \pm 4.4$ \\
\hline & After & $5.9 \pm 5.4$ & $157.6 \pm 15.5$ & $98.4 \pm 3.9$ \\
\hline
\end{tabular}

$\mathrm{PIPP}=$ Premature infant pain profile; $\mathrm{PR}=$ Pulse rate. 
Table 3. Results of Univariate Generalized Estimating Equation Analysis of the Premature Infant Pain Profile, Pulse Rate, and Oxygen Saturation

\begin{tabular}{|c|c|c|c|c|c|c|c|}
\hline \multirow{2}{*}{ Variables } & \multirow{2}{*}{ Categories } & \multicolumn{2}{|c|}{ PIPP } & \multicolumn{2}{|c|}{ PR } & \multicolumn{2}{|c|}{ Oxygen saturation } \\
\hline & & Estimate & $p$ & Estimate & $p$ & Estimate & $p$ \\
\hline \multirow[t]{2}{*}{ Group (distilled water) } & Human milk & -0.44 & .517 & -4.03 & .238 & -1.10 & .150 \\
\hline & Sucrose & 0.98 & .132 & 0.01 & .998 & -0.18 & .837 \\
\hline \multirow[t]{2}{*}{ Time (baseline) } & During & 10.84 & $<.001$ & 14.39 & .002 & -3.68 & $<.001$ \\
\hline & After & 2.94 & .003 & 5.75 & .074 & -0.44 & .638 \\
\hline \multirow{4}{*}{$\begin{array}{l}\text { Interaction } \\
(\text { distilled water } \times \text { baseline })\end{array}$} & $\mathrm{HM} \times$ during & 1.09 & .371 & 0.06 & .993 & 0.83 & .525 \\
\hline & $\mathrm{HM} \times$ after & 0.06 & .956 & 8.75 & .138 & 0.65 & .542 \\
\hline & Sucrose $\times$ during & 0.16 & .886 & 3.58 & .551 & 2.35 & .047 \\
\hline & Sucrose $\times$ after & -0.94 & .450 & 2.85 & .526 & 0.04 & .976 \\
\hline \multicolumn{2}{|l|}{ Gestational age } & -0.85 & .007 & -3.52 & .210 & -0.28 & .356 \\
\hline \multicolumn{2}{|l|}{ Post-conceptional age } & 1.13 & .004 & 4.20 & .271 & 0.43 & .215 \\
\hline \multicolumn{2}{|l|}{ Apgar score at $1 \mathrm{~min}$} & 0.43 & .238 & 0.93 & .675 & -0.95 & $<.001$ \\
\hline \multicolumn{2}{|l|}{ Apgar score at $5 \mathrm{~min}$} & -0.82 & .037 & 0.86 & .664 & 1.25 & $<.001$ \\
\hline
\end{tabular}

PIPP=Premature infant pain profile; $\mathrm{PR}=$ Pulse rate; $\mathrm{HM}=$ Human milk.

among the three groups of infants. Compared to baseline, PIPP, PR, and oxygen saturation significantly changed during the examination $(p<.001, p=.002$, and $p<.001$, respectively). The PIPP score remained elevated after the examination $(p=$ .003). Conversely, PR and oxygen saturation recovered to baseline values 2 minutes after the examination. Oxygen saturation was significantly higher during the procedure in the $24 \%$ sucrose group than in the control group $(p=.047)$. The PIPP score was more likely to be elevated in infants with younger gestational age at birth, higher post-conceptional age, or a lower Apgar score at 5 minutes $(p=.007, p=.004$, and $p=$ .037, respectively) (Table 3).

\section{DISCUSSION}

The current study found that the administration of a pacifier dipped in either $24 \%$ sucrose or human milk did not significantly reduce the pain associated with ROP examinations in preterm infants, compared to distilled water. This could have been the result of the amount of solution that was administered to infants via the pacifier. The exact amount of each solution was not measured in this study; however, it was likely approximately $0.1 \mathrm{~mL}$ based on the report of Mokhnach et al. [5]. In many studies supporting the pain-relieving effects of sucrose or human milk, at least $0.2 \mathrm{~mL}$ to $2 \mathrm{~mL}$ of solution was administered $[9,15,19,20]$. In addition, a single dose of the solution was administered just once 2 minutes prior to the examination; thus, its effect may not have lasted long enough to span the $2 \sim 3$ minutes of the examination. Mokhnach and colleagues [5] recommended administering three separate doses of sucrose, $0.1 \mathrm{~mL}$ each, during ROP examinations in order to ensure that the effects would last for 6 minutes. Further research is needed to test the pain-relieving effects of multiple doses of either human milk or sucrose.

This study found that the ROP examinations significantly changed the behavioral status, $\mathrm{PR}$, and oxygen saturation levels of the preterm infants. On average, infants' pain scores increased by 10 points from baseline during the examinations and remained 3 points higher than baseline scores even 2 minutes after the examinations. The severe pain (indicated by a PIPP score of $>13$ ) [18] related to the ROP examinations in our study is consistent with that found in other studies $[12,13,16]$. Despite the administration of topical anesthetic eye drops prior to the ROP examinations, following common clinical practice [1], preterm infants still require treatments to relieve pain during these examinations and to help them quickly recover afterwards. However, PR and oxygen saturation recovered to baseline levels by 2 minutes after the examinations, from the conditions of increased PR and decreased oxygen saturation during the examinations. In the study by Ou-Yang and colleagues [21], preterm infants undergoing heel pricks did not show changes in PR or oxygen saturation. This may have been related to the brevity of the heel prick procedure or the large amount $(5 \mathrm{~mL})$ of human milk or $25 \%$ glucose administered.

The relatively long duration of ROP examinations may induce greater levels of pain that exhibit different manifestations. For example, infants subjected to an ROP examination have more apneic events with concordant oxygen desaturation following the examination than prior to the examination [22]. Interestingly, the preterm infants in our study receiving $24 \%$ sucrose had a $2.5 \%$ higher oxygen saturation than those in the other groups. Because ROP examinations are necessary 
for infants born prior to 32 gestational weeks and who weigh less than 1,500 $\mathrm{g}[3,23]$, these infants are also at a higher risk of chronic respiratory problems. Our results suggest that sucrose may have a beneficial effect in terms of improving the oxygenation status of these infants during eye examinations in the NICU.

Younger and sicker preterm infants were more likely to have higher pain scores during ROP examinations in this study, which is consistent with the results of the recent study conducted by Collados-Gomez and colleagues [15]. They also showed that sucrose had a greater analgesic effect on infants born at a gestational age less than 28 weeks than it did in infants born after 28 weeks of gestation. However, Williams and colleagues [23] found that the behavioral response to pain was reduced in young and sick preterm infants. Both gestational age at birth and post-conceptional age should be considered when assessing infants' behavioral responses to pain, as well as their dose requirements for pain relief.

Bolye et al. [24] recommended using a pacifier in infants undergoing ROP examinations based on their finding that using a pacifier with or without $33 \%$ sucrose led to no significant difference. Gao et al. [25] also reported that non-nutritive sucking alone had a similar pain-reducing effect in preterm infants to that of sucrose in repeated heel lances, although the combination of non-nutritive sucking and sucrose was recommended to better manage ROP-related pain in infants compared to either management strategy used alone [25]. The abovementioned studies emphasized the benefits of pacifier use for pain relief in preterm infants. In our study, topical anesthetic, swaddling, and pacifier provision were used for all three groups of infants during the ROP examinations as the standard of care; however, our study did not find support for the hypothesis that soaking the pacifier in human milk or $24 \%$ sucrose would yield pain relief. The ideal method of administration and the duration of analgesia should also be investigated.

All participants were recruited at a single medical center, limiting the generalizability of the study results. Although the solution provided was not quantified before administration to individual infants, it can be assumed to be $0.1 \mathrm{~mL}$ [5]. Furthermore, this study included a small sample size, which may have limited the power of the experiment. Further research is needed to compare the pain-relieving effects of sucrose and human milk using a larger sample size.

\section{CONCLUSION}

Although ROP examinations are critical for the prevention of ROP-related vision loss in preterm infants, it is a painful and uncomfortable procedure. The application of a pacifier dipped in either human milk or $24 \%$ sucrose solution did not significantly reduce pain in preterm infants undergoing their first ROP examinations. However, a pacifier dipped in a $24 \%$ sucrose solution may improve the oxygen saturation level of preterm infants during ROP examinations. Further research is needed to investigate the pain-relieving effects of multiple applications of a pacifier soaked in either human milk or $24 \%$ sucrose during ROP examinations.

\section{Conflict of interest}

No existing or potential conflict of interest relevant to this article was reported.

\section{REFERENCES}

1. Lim Y, Godambe S. Prevention and management of procedural pain in the neonate: An update, American Academy of Pediatrics, 2016. Archives of Disease in Childhood Education and Practice Edition. 2017;102(5):254-256.

https://doi.org/10.1136/archdischild-2016-311066

2. Committee on Fetus and Newborn and Section on Anesthesiology and Pain Medicine. Prevention and management of procedural pain in the neonate: An update. Pediatrics. 2016;137(2):e20154271. https://doi.org/10.1542/peds.2015-4271

3. Bashinsky AL. Retinopathy of prematurity. North Carolina Medical Journal. 2017;78(2):124-128.

https://doi.org/10.18043/ncm.78.2.124

4. Sun X, Lemyre B, Barrowman N, O'Connor M. Pain management during eye examinations for retinopathy of prematurity in preterm infants: A systematic review. Acta Paediatrica. 2010;99(3): 329-334. https://doi.org/10.1111/j.1651-2227.2009.01612.x

5. Mokhnach L, Anderson M, Glorioso R, Loeffler K, Shinabarger K, Thorngate L, et al. NICU procedures are getting sweeter: Development of a sucrose protocol for neonatal procedural pain. Neonatal Network. https://doi.org/10.1891/0730-0832.29.5.271

6. Disher T, Cameron C, Mitra S, Cathcart K, Campbell-Yeo M. Painrelieving interventions for retinopathy of prematurity: A metaanalysis. Pediatrics. 2018;142(1):e20180401.

https://doi.org/10.1542/peds.2018-0401

7. Mangat AK, Oei JL, Chen K, Quah-Smith I, Schmölzer GM. A review of non-pharmacological treatments for pain management in newborn infants. Children. 2018;5(10):E130.

https://doi.org/10.3390/children5100130

8. Stevens B, Yamada J, Campbell-Yeo M, Gibbins S, Harrison D, Dionne K, et al. The minimally effective dose of sucrose for procedural pain relief in neonates: A randomized controlled trial. BMC Pediatrics. 2018;18(1):85.

https://doi.org/10.1186/s12887-018-1026-x

9. Kristoffersen L, Malahleha M, Duze Z, Tegnander E, Kapongo N, 
Støen R, et al. Randomised controlled trial showed that neonates received better pain relief from a higher dose of sucrose during venepuncture. Acta Paediatrica. 2018;107(12):2071-2078.

https://doi.org/10.1111/apa.14567

10. Gao H, Gao H, Xu G, Li M, Du S, Li F, et al. Efficacy and safety of repeated oral sucrose for repeated procedural pain in neonates: A systematic review. International Journal of Nursing Studies. 2016;62:118-125. https://doi.org/10.1016/j.ijnurstu.2016.07.015

11. Ribeiro LM, Castral TC, Montanholi LL, Dare MF, Silva AC, Antonini SR, et al. Human milk for neonatal pain relief during ophthalmoscopy. Revista da Escola de Enfermagem da USP. 2013;47 (5):1039-1045. https://doi.org/10.1590/s0080-623420130000500005

12. Rosali L, Nesargi S, Mathew S, Vasu U, Rao SP, Bhat S. Efficacy of expressed breast milk in reducing pain during ROP screening--A randomized controlled trial. Journal of Tropical Pediatrics. 2015; 61(2):135-138. https://doi.org/10.1093/tropej/fmu073

13. Dolgun G, Bozlak S. Effect of nonpharmacologic pain control during examination for retinopathy of prematurity. Journal of Obstetric, Gynecologic, and Neonatal Nursing. 2017;46(5):709-715. https://doi.org/10.1016/j.jogn.2017.06.008

14. Desai S, Nanavati RN, Nathani R, Kabra N. Effect of expressed breast milk versus swaddling versus oral sucrose administration on pain associated with suctioning in preterm neonates on assisted ventilation: A randomized controlled trial. Indian Journal of Palliative Care. 2017;23(4):372-378.

https://doi.org/10.4103/ijpc.Ijpc_84_17

15. Collados-Gómez L, Ferrera-Camacho P, Fernandez-Serrano E, Camacho-Vicente V, Flores-Herrero C, García-Pozo AM, et al. Randomised crossover trial showed that using breast milk or sucrose provided the same analgesic effect in preterm infants of at least 28 weeks. Acta Paediatrica. 2018;107(3):436-441.

https://doi.org/10.1111/apa.14151

16. Sener Taplak A, Erdem E. A comparison of breast milk and sucrose in reducing neonatal pain during eye exam for retinopathy of prematurity. Breastfeeding Medicine. 2017;12(5):305-310. https://doi.org/10.1089/bfm.2016.0122

17. Francis K. What is best practice for providing pain relief during retinopathy of prematurity eye examinations? Advances in Neonatal
Care. 2016;16(3):220-228.

https://doi.org/10.1097/anc.0000000000000267

18. Stevens B, Johnston C, Petryshen P, Taddio A. Premature infant pain profile: Development and initial validation. The Clinical Journal of Pain. 1996;12(1):13-22.

19. Dilli D, İlarslan NE, Kabataş EU, Zenciroğlu A, Şimşek Y, Okumuş N. Oral sucrose and non-nutritive sucking goes some way to reducing pain during retinopathy of prematurity eye examinations. Acta Paediatrica. 2014;103(2):e76-79.

https://doi.org/10.1111/apa.12454

20. O'Sullivan A, O'Connor M, Brosnahan D, McCreery K, Dempsey EM. Sweeten, soother and swaddle for retinopathy of prematurity screening: A randomised placebo controlled trial. Archives of Disease in Childhood Fetal and Neonatal Edition. 2010;95(6):F419-422. https://doi.org/10.1136/adc.2009.180943

21. Ou-Yang MC, Chen IL, Chen CC, Chung MY, Chen FS, Huang HC. Expressed breast milk for procedural pain in preterm neonates: A randomized, double-blind, placebo-controlled trial. Acta Paediatrica. 2013;102(1):15-21. https://doi.org/10.1111/apa.12045

22. Mitchell A, Green A, Jeffs D, Roberson P. Physiologic effects of retinopathy of prematurity screening examinations. Advances in Neonatal Care. 2011;11(4):291-297.

https://doi.org/10.1097/ANC.0b013e318225a332

23. Williams AL, Khattak AZ, Garza CN, Lasky RE. The behavioral pain response to heelstick in preterm neonates studied longitudinally: Description, development, determinants, and components. Early Human Development. 2009;85(6):369-374. https://doi.org/10.1016/j.earlhumdev.2009.01.001

24. Boyle EM, Freer Y, Khan-Orakzai Z, Watkinson M, Wright E, Ainsworth JR, et al. Sucrose and non-nutritive sucking for the relief of pain in screening for retinopathy of prematurity: A randomised controlled trial. Archives of Disease in Childhood Fetal and Neonatal Edition. 2006;91(3):F166-168.

https://doi.org/10.1136/adc.2005.087668

25. Gao H, Li M, Gao H, Xu G, Li F, Zhou J, et al. Effect of non-nutritive sucking and sucrose alone and in combination for repeated procedural pain in preterm infants: A randomized controlled trial. International Journal of Nursing Studies. 2018;83:25-33.

https://doi.org/10.1016/j.ijnurstu.2018.04.006 